\title{
La Radioterapia en los tumores de los niños
}

\author{
Dr. Jorge Mella Guerra*
}

Desde el descubrimiento de las radiaciones ionizantes, en 1906 el radiun por las esposos Curie y en 1898 los rayos $\mathrm{X}$ por Becquerel $\mathrm{y}$ Roentgen, éstas se han empleado en la medicina, en nuúltiples formas y en un sinnúmero de lesiones y afecciones del organismo. En la omcología pediatrica, su aplicatión ha ido attmentando y en esta última década sus indicaciones son prícticamente bien precisas.

Antes de entrar a analizar la clínica de las radiaciones, se expondrá algunas de las propiedátes biológicas, con algunos concejtos que el clinico clebe tener presente.

Las radiaciones jonizantes son elementos que alteran y desiruyen cualquier organismo viviente. Qué se entiende por muerte celular por raciacioness. Muerte oclular jor acliaciones es la destrucción que produce la radiación ionjzante a nivel del sistema de multiplicación celulitr. Est. alteración de la multiplicación celular puede ser inmediatal, proluciéndose la lisis celular o prescntarse en la 2a, 3 sa $^{\circ} 4^{\text {a }}$ generación. De este fenómeno, nace el concepto rle dosis letal, que es aquella cantidad cle irradiación ionizarte necesaria para producir la wuerte celular y el de dosis sub-letal, - que es aquella cantidad de racliación que altera el metabolismo celular, sin llegar a producir la destrucción del mecanisno de reproducción o multiplicación celular.

A comienzos del Siglo $\mathrm{xx}$, Bergonie y Tribondeau, investigadores franceses, observaron que aquellos tejidos cuyas células se multiplican muy tápidamente, eran más sensibles a las

* Fundación A. López Pérez. Santiago. radiaciones, que aquellos tejidos cuyas células st: multiplican mís lentamente; Jlevando este hecho al ampo de la histopatologia, se está bleció que los tejiclos indiferenciadlos o anaplásticos eran nuás radiosensibles que los diferenciados o macluros.

La radiobiología explica la ley de Bergonie y Tribondeau, a nivel del ciclo celular observánclose que las ratlaciones ionizantes actúan

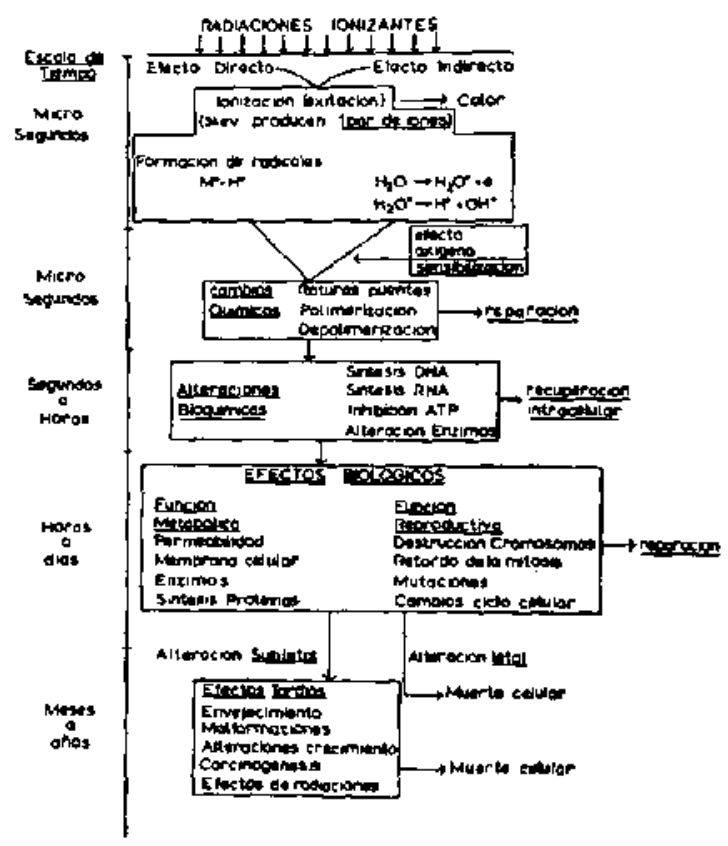

en fomma predominantemente, a nivel de la fase " $\mathrm{H}$ ", de división celular $y$ en la parte inicial de la fase " $\mathrm{S}$ ", de sintesis cle los ácidos nucleicos. Por lo tanto, aquellas células que presentan una fase $\mathbf{G l}$, o: 60 o $\mathbf{G}^{4} 2$, con tiempo de evolución muy largos, serán más ra- 
diorresistentes. que aquellas que tienen unat fase 61, o G0, o G2 con tiempo cle cvolu. ción más cortos. En otras palabras, podemos establecer, que mientras más corto son los períodos $\mathrm{Gl}$ y/o G2, más posibiliclades tencltán las radiaciones para actuar a nivel de la fase " $M$ " de división celular y de la fase inicial de lat sintesis de los ácitos nuclejcos. De la acción de las radiaciones a nivel del ciclo celular, so prede establecer el concepto de radiosensibili. dad: mientras más incliferenciado es un tejicts, tan to más sensible es a las radiaciones; es im concepto de tipo celular, diferente al concepto de radiocurabilidad, que es un análisis del efecto de las radiaciones solbre la region a tratar, en este caso, sobre el tumor, y el organjsiro en general. Un tumor puede ser muy radiosensible, pero poco radiocurable: ej. el reliculo. sarconia, el linfosarcoma, el tumor de Wilmus, etc. $O$ al revés, poco radiosensible; pero radiocurable: ej. el atenocarcinoma el retinoblastoma, el liposarcoma, etc.

Otro fenómeno importante que nos ha demostrado la radiobiología, en el sentido de racliosensibilidad, es el "efecto oxígeno". Para que las radiaciones ionizantes actuen a nivel relular, es necesario la presencia de agua, $\mathrm{H}_{2} \mathrm{O}$, ya que las radiaciones a través de su mecanis. mo indirecto, descompone el agua en radicales altanente activos como el $\mathrm{OH}^{-}$y el $\mathrm{H}^{+}$, los que irían a producir posteriormente las alteraciones celulares correspondientes. Para que esta accion sc lleve a efecto, es necesario un anbiente saturado de oxígeno, olservándose que aquellos territorios mal oxigenatos, clonde cxiste una baja presión de oxigeno, son territorios altamente radiorresistentes. La aplicación de este fenómeno en la clínica, es de mucha importancia, y para que el efecto oxigeno se manifieste en su más alto rendimiento es necesario tomar en cuenta en el análisis de un tratamiento radiante la presencia de necrosis, de tejidos mal vasculariados, de intervenciones quirúrgicas previas, del tamaño del tuinor, etc.

Descle el punto de vista de la acción de las radiaciones sobre el períorlo de presíntesis de los ácidos nucleicos y sobre toxlo a nivel del período " $M$ " de división celular, se producen alteraciones cromosómicas con consecuencias somáticas y en especial de tipo genéticass que se manilestarian no sólo en la primera genera. cion. sino que en unt alla proporción estats mutaciones se presentarian a posteriori, en la 2a, ya ${ }^{\text {a }} 4$ generación. Este hecho nos enseña a evitar el mal empleo de las radiaciones ionizantes; mientras menos irradiación reciba un organiumo, tanto mejor será para su desarrollo, como para su descendencia.

En la clínica, el objetivo de la radioterapia en el tratamiento de los tumores malignos, ts ir a lit destrucción del tejiclo canceroso, conservando los tejidos sinos. En el tratamiento de los tumores de los niños, este principio rara vez es aplicable sin comprometer el tejiclo noble. Al intentar de administrar una canlidad de radiación para destruir la celula cancerosa, el tejido sano o los órganos en la zona afectada, detienen su crecimiento o su función. Por otto bado, al administrar una dosis de radiación insuficiente, de tal manera que no comprometiera el crecimiento sle los tejidos normales o no alterara la buena función de un organo, esta cantidad de irradiación, seria insuficiente para destruir un tumor canceroso. El graclo de alteración somática clepenclería de la edad del niño y de la zona que reciba el tratamiento, mientras menos edad, más problemas en el desarrollo del niño se obsetvará.

El conocimiento de las secuel-as de la irtadiación en el tratamiento de los tumores malignos del niño, es fundamental para indicar $y$ planificar un tratamicnto. Igualmente es importante un análisis acusioso de la enfermedad, pira poder meditar y sobrepesar, el resul. tado clínico del tratamiento, frente a las posibles secuelas en un futuro no lejano. Este concepto de secuela, tanto somática como genética, es uno de los elementos más importanies cn la progranación cle los tratamientos de los cáncetes de los niños, heclo que diferencia fundamentalmente al tratamiento actínico del adulto donde el elemento secuela casi se desprecia. Por esta razón, los equipos radiantes, las técnicas y los fraccionanientos de los tratamientos de radioterapia empleados en el adulto o en el postadolescente, no pueden ser aplicados indiscriminadamente en el trata- 
miento de las enfermedarles neoplásicas ded niño.

Para evitar en lo posible llegar a producir sccuslis de importancia, se ha estatblecido que cardat clad tiene sus indicaciones en cuanto a canticlad de irradiación a aclministrar, por Ej: a nivel del sistema nervioso central las thosis es de 8.000 rads. a 5.000 rads. (Tabla No l). En el tumor de Wiluns está indicato

Tablat No 1

SISTFHA NERIOSO CENTRAI.

Doxis de ratlisteratpia y erfad

\begin{tabular}{rr}
\hline Edad & Dosis tumetr \\
\hline menor de 2 athos & 3.000 rats \\
$2-5$ años & 3.750 rads \\
$5-12$ años & 450 rads \\
sobre 12 años & 5.000 rails \\
\hline
\end{tabular}

1.800 rads, a un niño de menos de 18 meses y hasta 4.000 rads., sobre una edad de 12 meses. (Tabla 2).

Tabla Ni 2

TLIMCOR I)E WVIL.MS

Dosis de racliotenapia post. op.

\begin{tabular}{|c|c|}
\hline Edad & Dosis tumor \\
\hline menor de 18 meses & l. $300(0-2,1(5)$ rakls. \\
\hline 19.90 uteses & $9.4(0) \cdot 3.000$ ladk. \\
\hline 31.40 nlescs & $3.000-3.500$ rads. \\
\hline 42 uncses o màs & $3.500-4.000 \mathrm{rads}$ \\
\hline
\end{tabular}

Además de la edad, es necesario tener presente la zona a tratar, protegiendo los órganos fundamentales con protectores de plomo (figura l) como ser: los pulmones, los riñones, Jos genitales, cuando se trata la enfermedad cle Hodgkin.

Analizando todas estas variables, tipo his. tológico, ubicación dcl tumor, su oxigenación, su tamaño, organos vitales que lo rodean, edad del paciente, etc., se programa un tratamiento radiante, de acuerdo a un sistema donde debe intervenir el radioterapeuta, el físico, el tec-
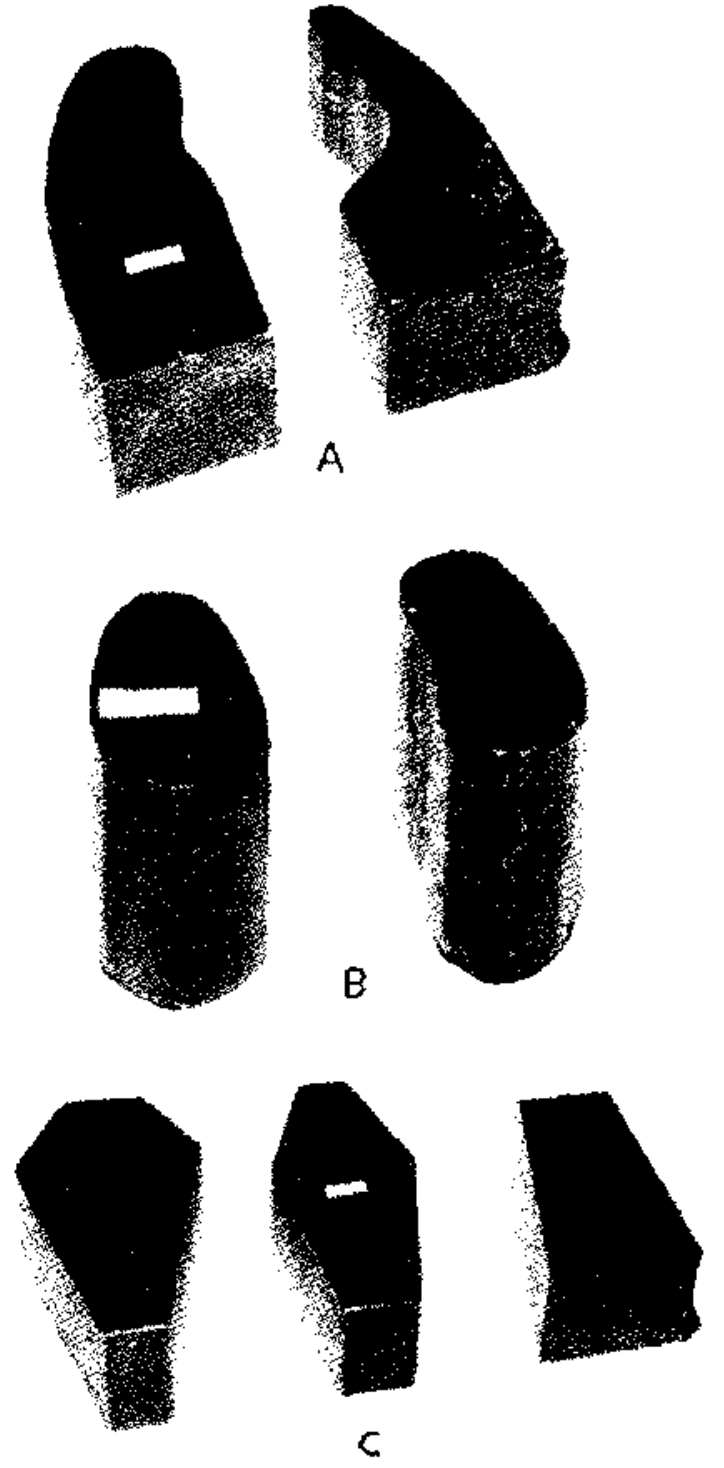

Figura 1

Proteclores the plono: A: pulnones: B: riôones; $C$ : genitites.

nólogo. para oblener, de esta manera un tratamiento eficar, con tun mínimo de secuelas (tabla 3).

Ahora bien, equé se entiende por radioterat pia en clínica?. Se entiencle por radioterapia en clínica al empleo de cualquier tijo de irracliación ionizante, en el tratamiento de una afeción determinadia. Existen, diferentes clases de radiaciones ionizantes entre las que tencmos: a) $\mathrm{La}$ radiación alfa, es un elemento de tipo corpuscular (núcleo de Helio). que dada su escasísima penetración no se enplea 
PROGRAMACION DE UN TRATAMIENTO BAPIANTE

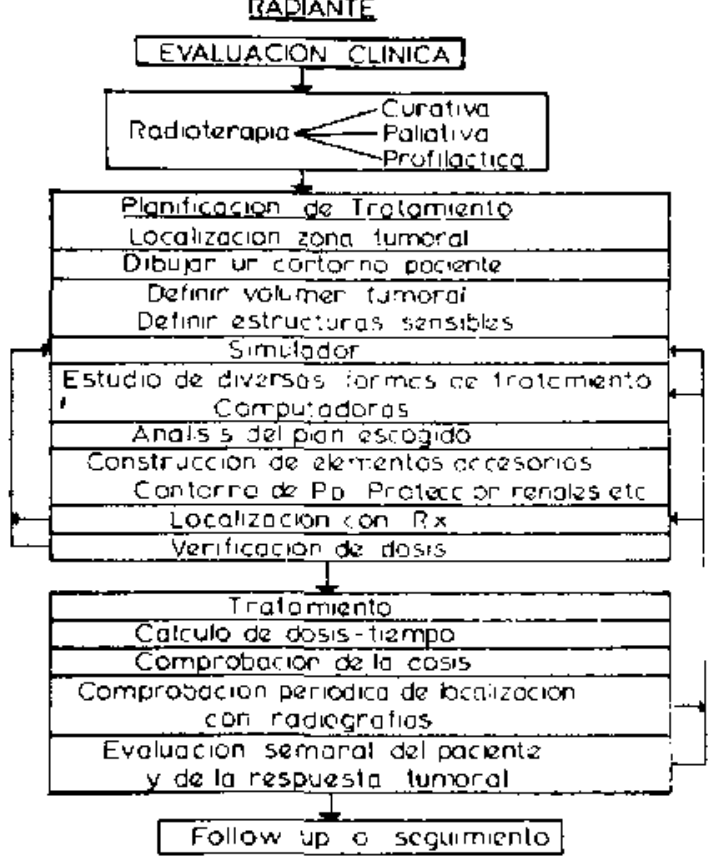

en estes cuadro de la cínica. b) La radiación beta, igualmente corpuscular; son electrones mucleares, de un poco mús de penctración que la radiación alla, esti representada especialmente por el cstroncio 90, y su cmpleo en la clínica es bien especifico en los lactantes que padecen de liemangionas civernosos, pitra evilar la irradiación de Jos cartílngos de crecimientos u otros órganos sensibles, dado que su penetración máxima es de 3 a 4 mum. c) lat radiacion gamat y los rayos $\mathrm{X}$, que son ondas elcctromancécicas de gran penetración, esti representada por el colsalto 60 , el cesieum 137 y los equipos comunmente llamados de raclioterapia.

Lats radiaciones se miden por uni unidad denominada roentgen, empleando la sigla " $r$ ", en honor al descubridor de los rayos X, Connaldo Roentgen. La cantidad de radiación que redibe un tumor se clemomina "rads", que es la (losis absorvirla por esc tejido (tabla 4).

Desde el punto de vista de la fuente radiante en relación al organismo, se tienen diferctstes tipos (le radioterapia (tabla. 5).

En la radioterapia externa, se tienen diferentes equipos de radioterapia, los que se iij " $\mathrm{r}$ ": boentgen aire

1) "rads": restitgen alssorvido en tuntor

lablia No:

TIPOS DE RAIIOTER APIA

i) Radioterapia Exicrua: Flemento radiatu[e at distam. cia del organismo ej: lomulsa Co.. aceleradors.

2) Radiotenapiat de Contaclo: Elemento radiante en

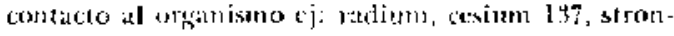
(j) 50 .

3) Raklioterapia Interua: Efentute radiante en el thganisino aj: vaclo 131-fiosforo, oro lise, cic.

catalogan de acucro a su potencia o kilovoltaje que esta relacionado con la longitud de onded de la racliación emitidiu (tabla 6). Al respecty es imprortante lacer presente, que en el capítulo de la oncología pecliátrica, la radiación que se cncuentra mís jndicada es la del rubro de los $3.000 \mathrm{Kv}$, prácticamente aquella que emite el isitopo radiactivo del colsalto fio, representado en el cquipo de radioterapia que so denomina bomba de cobalto. Esta radiación tiene la característica de que la absorción . nivel del hueso es mucho menor que rualyuicra otra isradiasión.

\section{Tilbla No: 6}

\section{TIPOS DE RADIOTERAPIA EXTERNA}

1) Radiolerapia superficial: epripe de chaoul J6) $\mathrm{Kv}-100 \mathrm{Kr}$

2) Ridioterapia Consencional: equipos de Ro prolunde (10) Kv $20(\mathrm{~K}) \mathrm{KY} 400 \mathrm{Kv}$

:3) Ratlienerapia Supersollaje: I.000 Kr.3.(50) $\mathrm{Kv}$ bomba de C.80 accleratores lincales

f) Radioncrapia Megavoltaje: aceTeratores fineales $3.000 \mathrm{KV}$ y nás Jetiltron Giclntró.1 Terapia de ncutrones 
En clínica estos elementos radiantes pueden emplearse asociados a otras terapias o en forma exclusiva según el cuadro adjunto (tatbla 7):

Tabla No ?

TIPOS DE RADIOTERAPIA CIINIC:S

a) Exclusivo: T. Ewing-Tinf, Hodgkit ctc

b) Preoperatorios: T. de Wilms. Neuroblatstomas etc.

c) Postoperatorios: 'T. de Wilus Neuroblastmas.

d) Asociada a Citontiticos: T. Wilms T. tejidos blanlos $T$. oseos Rutinoblastomas, Linfomas etc.

En cealquier terapia oncologica el fin terapéutico delye ser manifcstacto. En la progra. mación cle tola terapia radiante debe quedar constancia si la radioterapia es a titulo cura. tívo paliativo o profíáctico (Tabla 8).

Tabla $\times 8$

TIPOS DE RADIOTERAPIA CLINICA

a) Gurativo: Tumor localizado $\mathrm{T}_{1}-\mathrm{T}_{2}$

b) Paliativo: Metóstasis centros inıportantes.

c) Profilictico: Irradiación SNC (Lcuccunia).

Reacciones y tolerancia de los tejidos nomales frente a las radiuciones:

Sistemn hematopoyético: los efectos de las ra. diacioncs en el sistema hematopoyćtico del nj. ño es semejante al de los aclultos: leucopsenia con compromiso de los linfocitos y de los leucocitos polimorfonuclcares, una baja de las plaquctas y por último alteración de la serie roja. Es necesario recalcar que la relación volumen a irradiar y organismo completo, en el niño es mucho mayor que en tl adulto. Además, dado las características propias de la oncología pediátrica, ]a radioterapia gencralmente se asocia a la quimioterapia citotóxica.

Sistema nervioso central: su tolerancia es semejante al ded adulto. La tolerancia está en relación al volumen a irradiar: un volumen pcqueño puedle tolerar 5.000 radls., en 6 semanas, todo el cerebro no más de 4.000 rads., en 6 semanas. La médula espinal condiciona su tolerancia en relación al largo: más de $20 \mathrm{cms}$. de longitud no pasar las 8.000 rads., en 5 a 6 semanas, menos de $20 \mathrm{cms}$. de longitud puede tolerar 4.500 racls., en 5 a 6 semanas.

Puhnones: se produce una neumonitis y su intensiclad dependerá de la cantidad de irra. diación que reciba. Se puede irradiar todo el tórax: pơr ej. en tumor de Wilms metastásico adnıinistrando una closis de 1.200 a 1.500 rads., en dos semanas asociado a actinomicina, sin producir alteraciones clínicas de importancia (I)Angio) .

Ritiones: en los niños no se ha estucliado su tokrancia a las radiaciones, sin embargo, on adultos Kunkler ha observarlo que con and closis de radiación de 1.700 rads., en 5 semanas no se presentan altcracione's, en cambio con 2.800 rads. en 5 semanis se prescnta lua nefritis actínica en un alto porcentaje de los casos.

Ozario y testiculos: no existe dosis permisible. Estos órganos deben ser totalmente protegidos o irradiados con dosis totales.

Fejido Oseo: Neuhauser y col. Iian establecido que dosis sobre las 2.000 rads., producen un retardo en cl crecimicnto óseo, independiente de la edad del niño.

Sarcoma for irradiacion: en la literatura se han infornado de casos aislados. Fn el departamento de radioterapia tel M. D. Anderson, Houston, se ban presentado hasta el año 1070 , 4 casos.

Cristalino: Merriman y Focht lian observado que cxiste una enorme diferencia en la sensibiliclad a las racliaciones deI cristalino, entre los áchulos y los nif́ros. En su serie la observado que los cristalinos de los niños menores de un año muestran mayor incidencia de catarilats y estas se desarrollan rapidamente. Además ha establecido que sobre la exlad de un año, la sensibilitad del cristalino es semejante al del adulto. Uná dosis de radiación cle 750 a 950 rads., en 3 semanas, en un paciente de 5 meses de edacl, éste tendría un $60 \%$ de posibilidades de desarrollar una catarata y en el $50 \%$ de éslos, la evolución es altamente progresiva. 
Por últino, después de analizar en forma tan somera, Jos elementos y los problemas de la radioterapia en el tratímiento de los tumores de los niños, es necesario recalcar, que el trabajo en equipo del cirujano, del quimioterapeuta, del anatomopatólogo, del inmunólogo y del radioterapeuta, es fundamental, para desarrollar y programar tratamientos oncológicos adecuados para cada exlad. Farber. en su monografía, es enfático en insistir, que la sobrevida de los niños tratados por un equipo de médicos especialistas, es mejor, tanto del punto de vista de tiempo, como de secuelas, que aquellos niños tratados en centros no especializados.
1. The Ratliobiology of Human Canecr Radiuturapy. J. Robert Andrews. M. D. Saunders Company-Philadelphia, 1 in68.

2. Textlook of Rikliotherapy. Cillbet Fletcler, s. cond Fatition. 1 ca \&: Feldiger, 1:179.

3. Clinical Pediatric Onculogy-Watarn W', Sutow, ML. by Company. 1973.

4. Radiation Onculagy: Williams T. Moss, Misby Com. rany, Fonth Edition, 1973.

5. Frontiers of Radiationtherapy and Oncology. J. M. Vaeth, S. Karger Base]. 1968. 